\title{
Correction to: A Bifactor Model of the Straightforward Attentional Control Scale
}

\author{
Matt R. Judah ${ }^{1,2}$ (D) Kevin G. Saulnier ${ }^{3} \cdot$ Nathan M. Hager $^{1,2} \cdot$ Nicholas P. Allan $^{3}$ \\ Published online: 18 November 2019 \\ (C) Springer Science+Business Media, LLC, part of Springer Nature 2019
}

Correction to: Journal of Psychopathology and Behavioral Assessment

https://doi.org/10.1007/s10862-019-09737-y

The Table 2 in the original version of this article contained mistakes. The entries 4 to 12 under the Items column are incorrect. The correct Table 2 is presented at the next page.

The online version of the original article can be found at https://doi.org/ 10.1007/s10862-019-09737-y

Matt R. Judah

mjudah@odu.edu

Kevin G. Saulnier

ks981615@ohio.edu

Nathan M. Hager

nhage005@odu.edu

Nicholas P. Allan

allan@ohio.edu

1 Department of Psychology, Virginia Consortium Program in Clinical Psychology, Old Dominion University, Norfolk, VA, USA

2 Virginia Consortium Program in Clinical Psychology, Norfolk, VA, USA

3 Ohio University, Athens, OH, USA 
Table 2 Straightforward Attentional Control Scale (ACSS) and factor loadings for the twofactor exploratory factor analyses
Items

ACS-S

General Nuisance

AC $\lambda$

1. It's very easy for me to concentrate on a difficult task when there are noises around.

2. When I need to concentrate and solve a problem, I do not have trouble focusing my attention.

3. When I am working hard on something, I do not get distracted by events around me.

4. My concentration is good even if there is music in the room around me.

5. When concentrating, I can focus my attention so that I become unaware of what's going on in the room around me.

6. When I am reading or studying, I am not easily distracted if there are people talking in the same room.

7. When trying to focus my attention on something, I do not have difficulty blocking out distracting thoughts.

8. I do not have a hard time concentrating when I'm excited about something.

9. When concentrating I ignore feelings of hunger or thirst.

10. I can quickly switch from one task to another.

11. It does not take me a while to get really involved in a new task.

12. It is easy for me to coordinate my attention between the listening and writing required when taking notes during lectures.

13. I can become interested in a new topic very quickly when I need to.

14. It is easy for me to read or write while I'm also talking on the phone.

15. I can easily carry on two conversations at once. $\quad .57 \quad .20$

16. I have an easy time coming up with new ideas quickly. $\quad .50 \quad .22$

17. After being interrupted or distracted, I can easily shift my $\quad .80 \quad .17$ attention back to what I was doing before.

18. When a distracting thought comes to mind, it is easy for me to shift my attention away from it.

19. It is easy for me to alternate between two different tasks.

20. It is easy for me to break from one way of thinking about something and look at it from another point of view.

\begin{tabular}{|c|c|c|c|}
\hline .60 & -.31 & .63 & -.32 \\
\hline .71 & -.27 & .78 & -.32 \\
\hline .74 & -.36 & .80 & -.39 \\
\hline .70 & -.22 & & \\
\hline .71 & -.35 & & \\
\hline .68 & -.28 & .73 & -.24 \\
\hline .75 & -.27 & .81 & -.23 \\
\hline .64 & -.11 & .69 & -.10 \\
\hline .44 & -.05 & & \\
\hline .67 & .33 & .71 & .38 \\
\hline .69 & .15 & & \\
\hline .68 & .22 & .72 & .26 \\
\hline .62 & .22 & .67 & .31 \\
\hline .60 & .22 & & \\
\hline .57 & .20 & & \\
\hline .50 & .22 & & \\
\hline .80 & .17 & .89 & .20 \\
\hline .75 & .01 & .82 & .05 \\
\hline .73 & .38 & .75 & .42 \\
\hline .66 & .23 & & \\
\hline
\end{tabular}

ACS-S Short

General Nuisance AC $\lambda \quad \lambda$

ג

